\title{
Exploiting an Ontological Model to Study COVID-19 Contagion Chains in Sustainable Smart Cities
}

\author{
Nemury Silega ${ }^{1, *(1)}$, Eliani Varén ${ }^{2}$, Alfredo Varén ${ }^{3}$, Yury I. Rogozov ${ }^{1}$, Vyacheslav S. Lapshin ${ }^{1}$ \\ and Skolupin A. Alekseevich ${ }^{1}$ \\ 1 Department of System Analysis and Telecommunications, Southern Federal University, \\ 347900 Taganrog, Russia; yrogozov@sfedu.ru (Y.I.R.); lapshin@sfedu.ru (V.S.L.); skorlupin@sfedu.ru (S.A.A.) \\ 2 Facultad de Ciencias y Tecnologías Informáticas, Universidad de las Ciencias Informáticas, \\ Habana 19370, Cuba; evaren@uci.cu \\ 3 Centro Provincial de Higiene, Epidemiología y Microbiología, Cienfuegos 55100, Cuba; \\ alfredo.varen@gmail.com \\ * Correspondence: silega@sfedu.ru
}

check for

updates

Citation: Silega, N.; Varén, E.; Varén,

A.; Rogozov, Y.I.; Lapshin, V.S.;

Alekseevich, S.A. Exploiting an

Ontological Model to Study

COVID-19 Contagion Chains in

Sustainable Smart Cities. Information

2022, 13, 40. https://doi.org/

$10.3390 /$ info13010040

Academic Editors: Haridimos

Kondylakis and Wade Trappe

Received: 13 November 2021

Accepted: 5 January 2022

Published: 14 January 2022

Publisher's Note: MDPI stays neutral with regard to jurisdictional claims in published maps and institutional affiliations.

Copyright: (c) 2022 by the authors. Licensee MDPI, Basel, Switzerland. This article is an open access article distributed under the terms and conditions of the Creative Commons Attribution (CC BY) license (https:// creativecommons.org/licenses/by/ $4.0 /)$.

\begin{abstract}
The COVID-19 pandemic has caused the deaths of millions of people around the world. The scientific community faces a tough struggle to reduce the effects of this pandemic. Several investigations dealing with different perspectives have been carried out. However, it is not easy to find studies focused on COVID-19 contagion chains. A deep analysis of contagion chains may contribute new findings that can be used to reduce the effects of COVID-19. For example, some interesting chains with specific behaviors could be identified and more in-depth analyses could be performed to investigate the reasons for such behaviors. To represent, validate and analyze the information of contagion chains, we adopted an ontological approach. Ontologies are artificial intelligence techniques that have become widely accepted solutions for the representation of knowledge and corresponding analyses. The semantic representation of information by means of ontologies enables the consistency of the information to be checked, as well as automatic reasoning to infer new knowledge. The ontology was implemented in Ontology Web Language (OWL), which is a formal language based on description logics. This approach could have a special impact on smart cities, which are characterized as using information to enhance the quality of basic services for citizens. In particular, health services could take advantage of this approach to reduce the effects of COVID-19.
\end{abstract}

Keywords: COVID-19; ontology; artificial intelligence

\section{Introduction}

Coronaviruses are a family of viruses found in certain animals, which become infection agents capable of causing serious diseases to humans through mutations, such as severe acute respiratory syndrome (SARS), Middle East respiratory syndrome (MERS) and SARSCoV2 discovered in China in 2019. SARS-CoV2 causes the COVID-19 disease. The World Health Organization (WHO) declared COVID-19 a world pandemic due to the high number of infections and deaths that this disease had caused around the world.

The high transmissibility of SARS-CoV2 and the existence of asymptomatic patients are factors that considerably complicate its containment. An exhaustive analysis of chains of contagion can provide new findings that may be useful for epidemiological and virological studies. For example, it might be possible to identify the symptoms in a chain of contagion and make a comparison with the symptoms in other chains. If notable differences are found, further analysis may be necessary to find the source of those differences, which could be either biological, social or environmental. For example, the differences might be due to either different variants of the virus or to different human behaviors.

Likewise, the analysis of contagion chains might help to identify the points where the virus starts to change its behavior or its effects. On the other hand, it could be possible 
to measure how long a chain has been active. This information could be useful to define strategies to reduce the options for contagion. However, the high number of infections means that the chains are long, which considerably slows down the work of researchers. This situation makes it difficult to perform an in-depth analysis. Moreover, the lack of tools to support the efforts of researchers means that their work cannot be carried out with the agility that the current pandemic demands. Therefore, this study addresses the problem of how to represent the information related to the chains of contagion of COVID-19 in such a way that its analysis is expedited and traits that contribute to characterizing the disease can be obtained.

Seeking approaches to deal with the aforementioned problem, we found that the adoption of ontologies is a suitable option to represent and analyze knowledge of different domains [1-7]. The semantic representation of information by means of ontologies enables the consistency of the information to be checked, as well as automatic reasoning to infer new knowledge.

This paper aims to present an ontological model to represent information regarding sick patients with COVID-19 to improve the analysis of the characteristics of the disease. The main contribution of this research lies in the fact that the analysis focuses fundamentally on the chains of contagion, unlike previous studies that have focused on other perspectives. The results of this approach could be useful for epidemiological work, especially in countries where rigorous monitoring of contagion chains has proven to be an effective strategy to contain the virus. Likewise, this ontology could be a useful instrument to support the work of researchers during experimental studies. The ontology was developed following a sound methodology that is recognized and widely used in the scientific community. In addition, its conditions were validated as a logical-formal system by means of reasoners.

The rest of the article is organized as follows. The next section presents the analysis of related works. The following section describes the ontological model developed to represent and analyze the information of patients with COVID-19 disease. A case study is presented next, which shows the applicability of the proposal. Finally, the conclusions of the article are presented.

\section{Related Works}

Through a literature review, we found some works that analyzed contagion chains to characterize COVID-19. For example, Wang studied the contagion chains in Tianjin, a Municipality in China [8]. This study yielded useful results and identified some features of the contagion chains, for example transmission from a family member constituted $42 \%$ of infections, usually at the end of the transmission chain. Schwarz analyzed a contagion chain related to an 11-year-old girl that was infected by her father [9]. Although this was a small contagion chain, it illustrated the advantages of this kind of analysis. Cheng covered information on contagion chains in an analysis of the two COVID-19 outbreak waves in Hebei Province, China [10]. Likewise, $\mathrm{Hu}$ et al. analyzed a chain of transmission involving 52 linked patients with COVID-19 in three separate hospitals in Wuhan [11].

Furthermore, we found other ontologies focused on representing specific information related to COVID-19. The Long COVID Phenotype Ontology (https://bioportal. bioontology.org/ontologies/LONGCOVID) (accessed on 20 September 2021) represents information to analyze long COVID-19 patients. The COKPME-COVID-19 Ontology (https:/ / bioportal.bioontology.org/ontologies/COKPME) (accessed on 20 September 2021) describes the relative and precautionary measures that can be put in place to control the spread of COVID-19. The COVID-19 Impact on Banking Ontology (https://bioportal. bioontology.org/ontologies/COVID19-IBO) (accessed on 20 September 2021) provides semantic information about the impacts of COVID-19 on the banking sector of India.

On the other hand, several studies applying ontologies in the domain of epidemiology were identified. We found two ontologies $[12,13]$ that represented and analyzed information about COVID-19. The first ontology aimed to represent COVID-19 patient data [13]. Despite the multiple benefits of this ontology, an analysis of contagion chains was outside of its 
scope. Likewise, the other ontology [12] included a huge number of classes, properties and relations to present and analyze COVID-19 information, although we did not find specifications to analyze contagion chains.

Other studies adopted ontological approaches to analyze infectious diseases or virus behavior in plants and animals [14-16]. The reviewed studies confirmed the potential of ontologies to represent and analyze information from the area of epidemiology or virological analysis. We believe that an ontology that can be used to analyze contagion chains could be useful in supporting the work of those researchers and specialists who are studying the behavior of this disease.

\section{Ontology for Representing and Analyzing Contagion Chains}

\subsection{Tools and Methodology}

Among the languages used to specify ontologies [17-22], the following stand out: Loom, CycL, Ontolingua, XML Schema, RDF (Resource Description Framework), RDF Schema (or RDF-S) and OWL 2 (Web Ontology Language). Slimani compared how the most popular languages manage to meet attribute criteria, facet, taxonomy, function and general issue criteria [21]. This analysis showed that OWL 2 had the best results, satisfying 20 of the 21 requirements.

OWL 2 is based on a logic model that makes it possible to define and describe concepts. The OWL 2 model is distinguished by its set of intersection, union and negation operators. It is based on a logic model that defines the concepts as they are described. Furthermore, the possibility of using reasoners allows the consistency of the represented models to be checked automatically. Taking into account its advantages, OWL 2 (hereinafter OWL) was adopted to represent our ontology.

To implement the ontology, the Protégé tool [23], which is an open-source and multiplatform editor developed at Stanford University, was used. It has a flexible and extensible architecture and is recognized as one of the most used tools for ontological engineering. Ontologies created in Protégé can be exported in various syntax, such as RDF / XML and $\mathrm{OWL} / \mathrm{XML}$. On the other hand, $\mathrm{FaCT}++$, Hermit and Pellet reasoners can be used in the inference process.

The adoption of a solid methodology is crucial to obtaining a quality ontology. Therefore, several methodologies were analyzed $[17,24,25]$. The development of the ontology presented in this research was guided by the methodology proposed by Noy and McGuinness, which has been widely adopted [26]. This is the most used or cited methodology for designing an ontology [27]. It includes the following steps: determine the domain and scope of the ontology, consider reusing existing ontologies, list the relevant terms of the ontology, define the classes and the class hierarchy, define the properties (called relationships or slots) of the classes, define facets or restrictions on slots or relationships and finally define instances.

\subsection{Results of the Ontology Development Process}

The execution of the methodology described in the previous section allowed us to obtain an ontology to describe and analyze COVID-19 contagion chains. Below the main results of the otology development process are described.

Step 1. Define the domain and scope of the ontology.

The ontology has the objective of providing the mechanisms to represent and analyze information regarding sick patients with COVID-19. Emphasis is placed on the chains of contagion to find information that could be useful in the studies that are developed on the disease. It is based on the premise that certain information on patients in isolation could be of little relevance, although if the analysis is carried out on a chain of contagion, conclusions of interest for the scientific community and for health and government authorities could be obtained. Patterns were identified, the presence of which might warrant a more detailed study to consider possible causes or implications. To specify the objective of the ontology, ten competency questions (CQ) were formulated: 
CQ 1. What patients belong to a chain of contagion?

CQ 2. Who has directly or indirectly infected another person?

CQ 3. Which chains include deceased people?

CQ 4. Which chains include people of only one sex exist?

CQ 5. Which chains include patients from different cities?

CQ 6. Which chains include pediatric patients?

CQ 7. Which are long chains?

CQ 8. Which chains exist in which all the members have recovered from the disease?

CQ 9. Which chains include only asymptomatic people?

CQ 10. How long has a certain chain been active?

\section{Step 2: Reuse existing ontologies.}

Although we did not find ontologies focused on representing the chains of contagion, we reused several concepts from different ontologies. We used the Bioportal repository (https://bioportal.bioontology.org/) (accessed on 10 September 2021) to search and analyze several ontologies. From the Semantic Science Integrated Ontology (https: / / bioportal.bioontology.org/ontologies/SIO) (accessed on 20 September 2021), we reused the Medical_Practicioner class, which is a subclass of the Person class. A Medical_Practicioner can be a Doctor or Nurse according to the role. We also adopted the Role class, Medical_Role class and the subclasses of Medical_Role. From the ontology Pediatric Terminology (https://bioportal.bioontology.org/ontologies/PEDTERM) (accessed on 20 September 2021), the hierarchy of the Child class was adopted. The identification of new concepts that could be reused to enhance our ontology is a future line of our research.

\section{Step 3: List the relevant terms of the ontology.}

From the review of the literature and the continuous monitoring of the information regarding the disease, a set of terms that are essential for modeling the ontology was identified. Among the most relevant terms were person, patient, chain of contagion, classification of patients, date of infection and symptoms, among others. These terms of reality constitute the basis for defining the structural elements of the ontology.

Step 4. Define the classes and class hierarchy.

In this step, we carried out an analysis to determine which of the terms defined in the previous step are modeled in the ontology as classes. Finally, 52 classes in the ontology were defined. The classes Person and Contagion_Chain are two of the main classes in the ontological model. Each of these classes subsumes other classes in order to characterize the individuals that compose it and offer analyses of interest. In Figure 1, the hierarchy of the class Person is shown. A diagnosed person with the disease can be classified as Person_Deceased, Person_Recovered or Patient_Active. An active patient can be classified as either Stable_Patient or Severe_Patient. In addition, a patient in a state of significant seriousness, with danger to their life, is classified as Critical_Patient. Additionally, the class Patient_Asymptomatic was included due to the high number of patients with this behavior and precisely because it is one of the characteristics that considerably influences the high transmissibility of the virus.

On the other hand, the hierarchy of the class Contagion_Chain that is shown in Figure 2 is also interesting. The defined classes in OWL (highlighted in the figure with the darker background) are a type of class to which necessary and sufficient conditions are defined. Therefore, a reasoner can automatically infer the individuals that belong to these classes. Taking advantage of these potentialities, several classes were defined to classify the contagion chains. The expressive richness of OWL 2 made it possible to define the necessary and sufficient conditions for various types of contagion chains, which will be explained in step 6.

\section{Step 5: Define properties.}

There are two types of properties in an ontology, object properties and data properties. Object properties are relationships between two individuals. In OWL, to restrict the classes that can use a property, the domain and range must be defined. For example, an individual of the class Contagion_Chain Is_Chain_Of individuals of the class Person. In this case, 
the property Is_Chain_Of has the classes Contagion_Chain and Person as the domain and range, respectively. Due to space limitations, only the properties of the classes Person and Contagion_Chain are included in Table 1, although the ontology includes 29 object properties in total. Furthermore, since object properties allow binary relationships to be established between individuals, each property has an inverse property. For example, the property Was_Directly_Infected_By has the inverse property Infected_Directly_To which allows one to establish that whether A Infected_Directly_To B then B Was_Directly_Infected_By A.

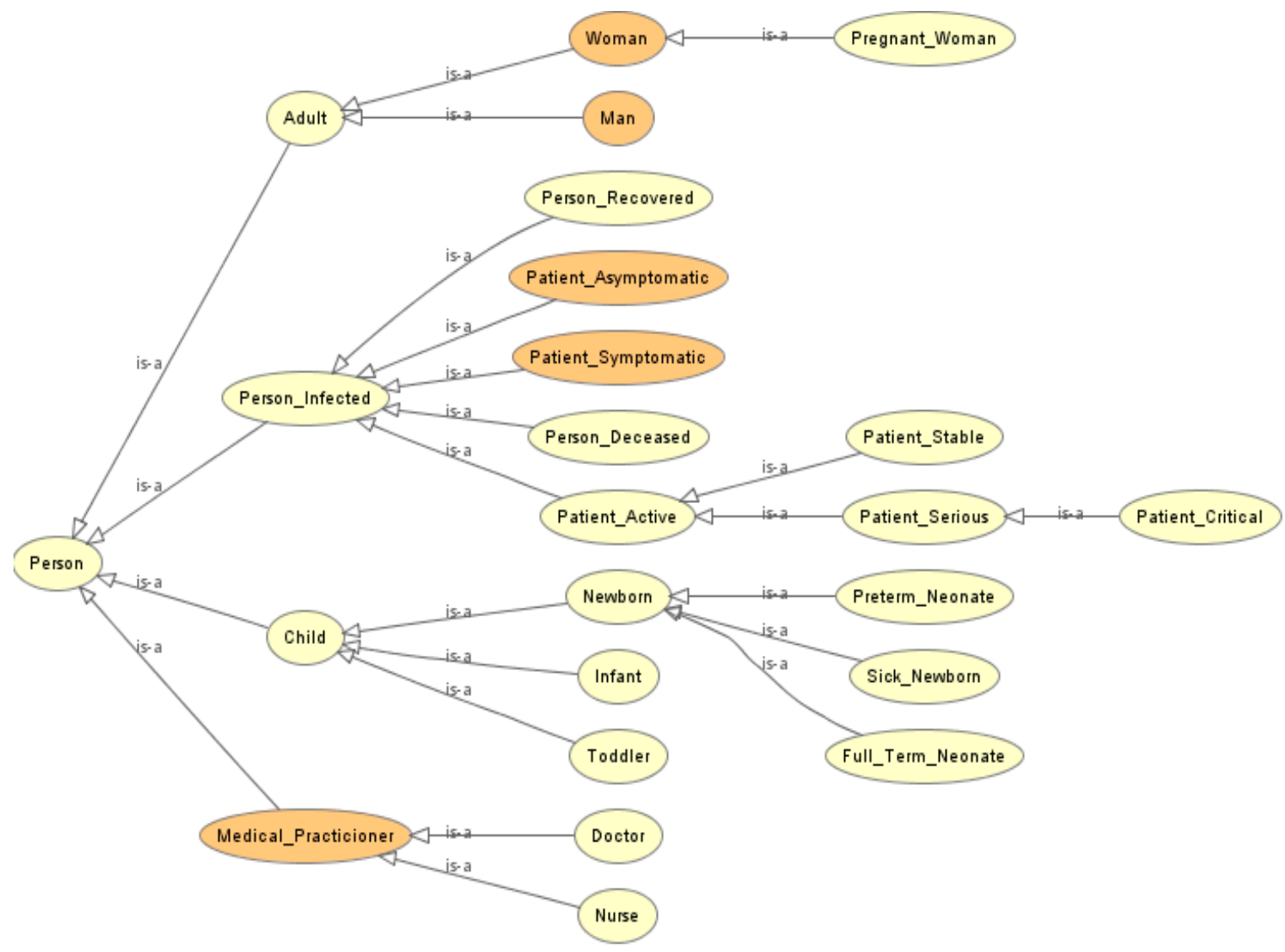

Figure 1. Hierarchy of the class Person.

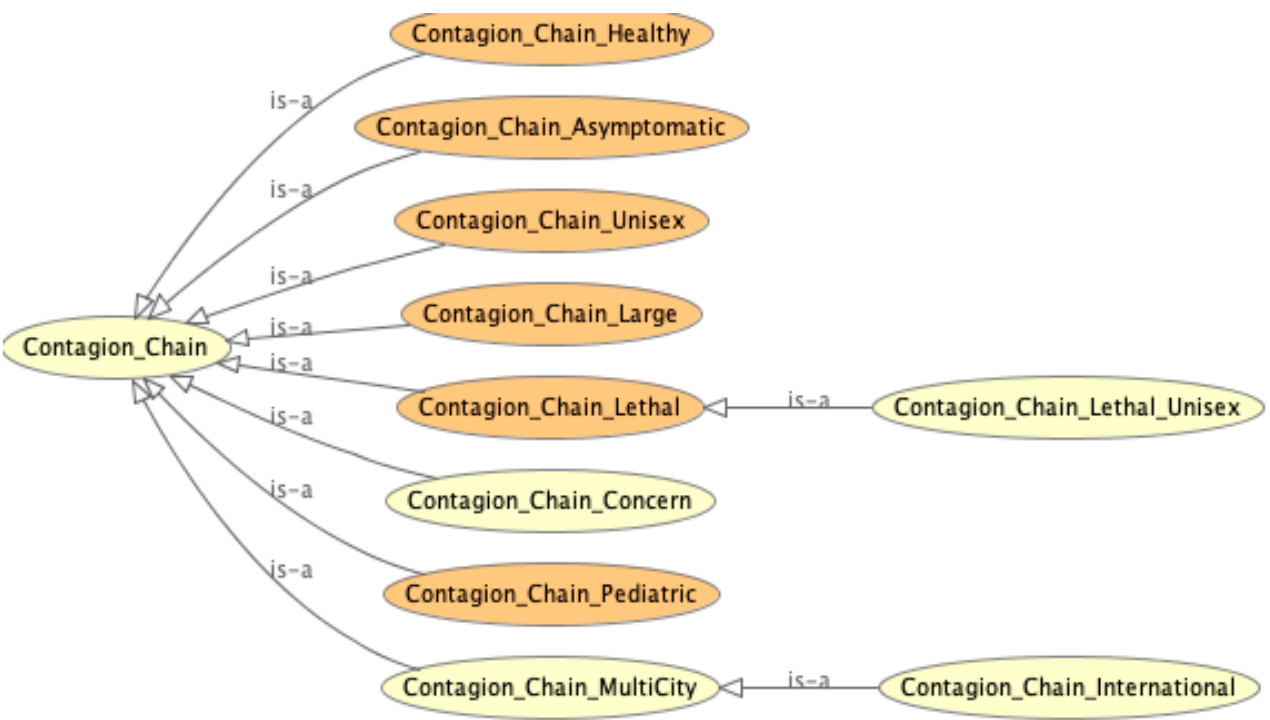

Figure 2. Hierarchy of the class Contagion_Chain. 
Table 1. Object properties of the classes Person and Contagion_Chain.

\begin{tabular}{ccc}
\hline Domain & Property & Range \\
\hline \multirow{2}{*}{ Contagion_Chain } & Is_Chain_Of & \\
& Has_First_Case & Person \\
& Has_Last_Case & \\
\hline Person & Was_Directly_Infected_By & Person \\
& Was_Directly_Infected_By & Sympton \\
Person & Has_Symptom & Municipality \\
Person & Lives_In_Municipality & Virus \\
Person & Is_Infected_With &
\end{tabular}

On the other hand, some data properties were also specified. Table 2 depicts the data properties of the classes Person and Contagion_Chain.

Table 2. Data properties of the classes Person and Contagion_Chain.

\begin{tabular}{ccc}
\hline Domain & Data Property & Range \\
\hline Contagion_Chain & Has_Date_Start & DateTime \\
& Has_Date_End & String \\
Person & Has_Name & DateTime \\
Person & Has_Birth_Date & DateTime \\
Person & Has_Contagion_Date & \\
\hline
\end{tabular}

\section{Step 6. Define restrictions.}

In this step, a set of existential, universal and cardinal constraints was defined. The set of restrictions for some defined classes are described below.

As a necessary and sufficient condition for the class Contagion_Chain_Unisex, it was defined that it could only have people of the same sex. In this case, a universal restriction was defined, characterized in OWL by the expression only, as shown in Figure 3a. For the class Contagion_Chain_Pediatric, it was defined that there should be at least one child in the chain. This was modeled from an existential constraint, identified in OWL by the expression some, as shown in Figure $3 \mathrm{~b}$.

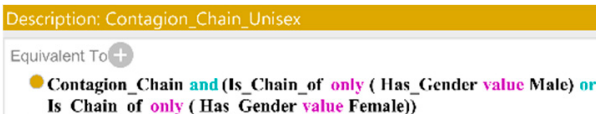

Contagion_Chain and (Is_Chain_of only (Has_C
Is Chain of only ( Has_Gender value Female)

(a)

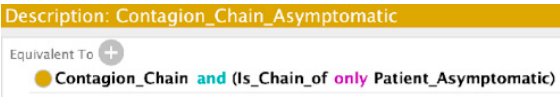

(c)

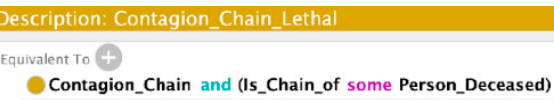

(e)

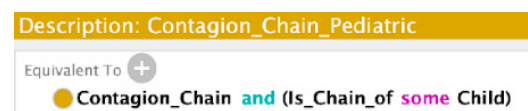

(b)

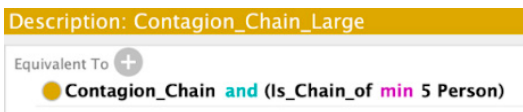

(d)

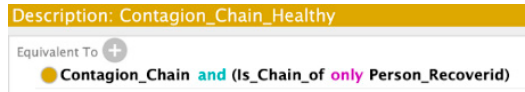

(f)

Figure 3. Definition of different types of Contagion_Chain: (a) Contagion_Chain_Unisex; (b) Contagion_Chain_Pediatric; (c) Contagion_Chain_Asymptomatic; (d) Contagion_Chain_Large; (e) Contagion_Chain_Lethal; (f) Contagion_Chain_Healthy. 
For the class Contagion_Chain_Asymptomatic, it was defined that all members of the chain should be asymptomatic. For this, a universal restriction was defined, as shown in Figure 3c. For the class Contagion_Chain_Large, a cardinal constraint was created, which defines that a chain must have at least 5 people, as illustrated in Figure 3d. This threshold was established as an example; in a real environment it would be higher. The Contagion_Chain _Lethal includes at least one deceased person among its members. Therefore, it was defined as an existential constraint, as shown in Figure 3e. Additionally, it was defined that in a Contagion_Chain_Healthy, all members are recovered people. Therefore, it was also defined by a universal relation, as shown in Figure $3 f$.

A Contagion_Chain_Multi_City has members of different cities. To identify the instances of the class Contagion_Chain_Multi_City, certain rules in Semantic Web Rule Language (SWRL) were stated. The time of a chain can be known through the dates of its first case and its last case. This was modeled with SWRL as well.

\section{Step 7. Define instances.}

In this step, individuals were defined for the specified classes and the corresponding axioms were established. The results of this step are explained in the next section by means of a case study. Through the case study, the applicability and usefulness of the developed ontology is demonstrated.

\subsection{Ontology Validation}

To validate the ontology, we checked that (a) it met the specifications as a formallogical system and (b) it satisfied the requirements for which it was created. To verify that the specifications as a formal-logical system are fulfilled, a reasoner is used. In this case, we used the reasoner Pellet, which confirmed that the ontology was consistent. It should be noted that the evaluation of the ontology is an iterative and progressive process. Throughout the life cycle of ontology development, we continually used the reasoner to verify the consistency of the ontology.

In addition, the last version of the ontology was evaluated using the OntOlogy Pitfall Scanner (OOPS!) (http:/ / oops.linkeddata.es/index.jsp) (accessed on 10 November 2021) [28]. After three iterations, all problems detected by OOPS were addressed. This evaluation helped us to detect and correct certain pitfalls in our ontology.

To demonstrate that the ontology satisfied the requirements for which it was created, we carried out a case study. In this study case, we verified that all competency questions were answered correctly by the ontology. This study case, described in the next section, also illustrates the usefulness and applicability of our ontology.

\section{Case Study}

To illustrate the usefulness and applicability of the ontology, a case study is presented below. The contagion chains shown in Figure 4 will be modeled in the ontology. Patient_1 infected to patients 2, 5 and 9. In the figure, the color of the number of patients shows their sex (red for male and blue for female). There are three chains: 1-2-3-4, 1-5-6-7-8 and 1-9-10-11-12. Additionally, as the caption of Figure 4 shows, some patient information is indicated. The modeling of this information in the ontology is illustrated below. Likewise, how the reasoning capacity of this ontological model is exploited to answer the competency questions defined in step 1 of the Section 3 is explained.

In Figure 5, different views of Protégé indicating how the information from Figure 4 was represented in the ontology are shown. Figure 5a shows the patients belonging to the class Man, Figure 5b illustrates that Patient_3 belongs to the class Patient_Deceased and Figure $5 \mathrm{~b}$ shows the patients that belong to the class Patient_Asymptomatic. Finally, Figures $5 \mathrm{c}$ and 5 e show the municipalities of Patient_2 and Patient_3, respectively.

In addition to the individual information for each patient, certain mechanisms to identify the contagion chains were defined. Through the property Directly_Infected_To, it is specified that Patient_1 infected to patients 2, 5 and 9. Subsequently, direct transmission of the rest of the patients can be established. After those statements, the reasoner is able 
to infer that Chain_1 contains patients 1, 2, 3 and 4, responding to CQ 1. In Figure 6a, the inference of the reasoner is shown (inferences in Protégé are shown with the yellow background). To achieve this inference, the property Infected_To is defined as transitive, exploiting the expressive richness of OWL. Furthermore, as a subtype of this property, Directly_Infected_To is defined. In this way, the traceability of all the people who indirectly infected a specific patient is achieved, as shown in Figure 6b for Patient_8.

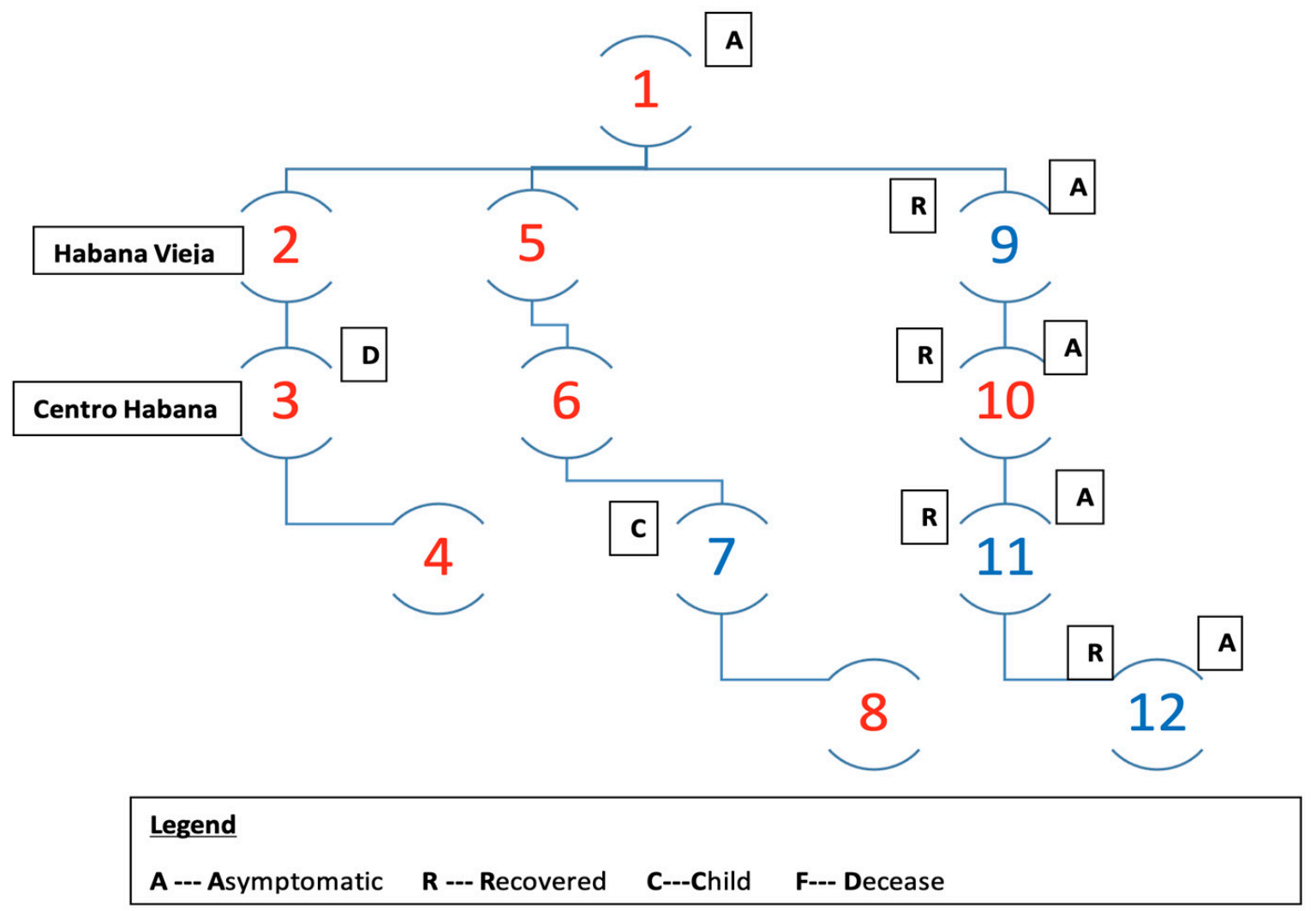

Figure 4. Example of contagion chains.

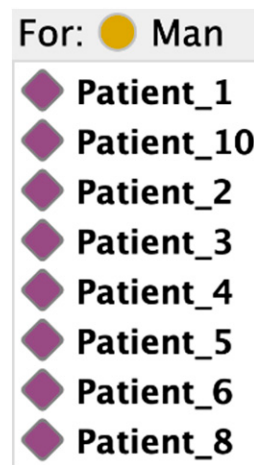

(a)

\section{For: Person_Deceased \\ Patient_3}

(b)

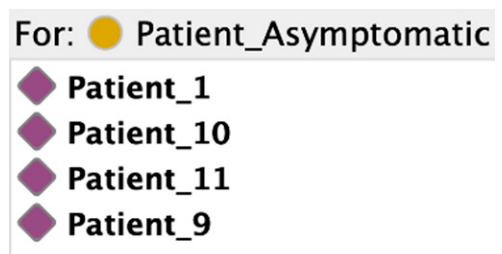

(d)

\section{Property assertions: Habana_Vieja}

Object property assertions

- Is_District_Of Patient_2

(c)

Property assertions: Centro_Habana
$\begin{aligned} & \text { Object property assertions } \\ & \text { Is_District_Of Patient_3 }\end{aligned}$

(e)

Figure 5. Representation of patient information: (a) Individuals of the class Man; (b) Individual of the class Person_Deceased; (c) District of Patient_2; (d) Individuals of the class Patient_Asymptomatic; (e) District of Patient_3. 


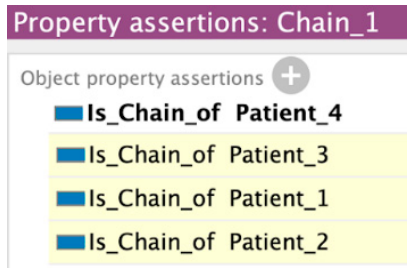

(a)

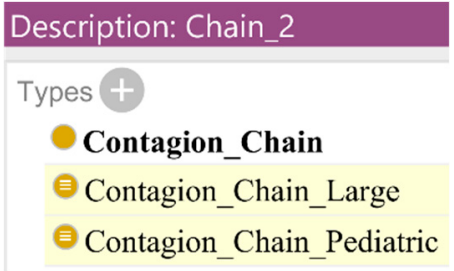

(d)

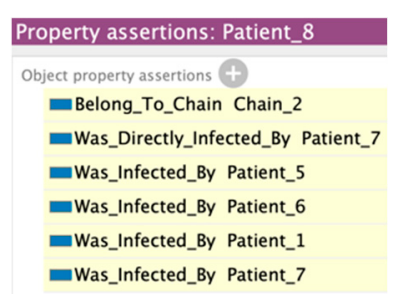

(b)

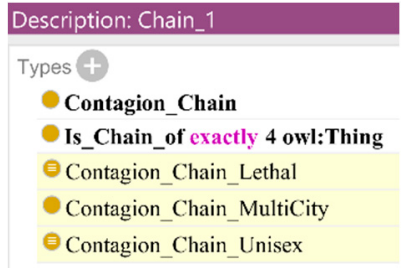

(c)

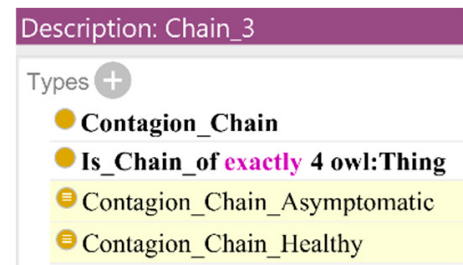

(e)

Figure 6. Inferences about the contagion chains: (a) Members of Chain_1; (b) Assertions of Patient_8; (c) Classifications of Chain_1; (d) Classifications of Chain_2; (e) Classifications of Chain_3.

Since all members of Chain_1 are male, the chain should be classified as Contagion_Chain_Unisex. After modeling it in ontology, as shown in Figure 5a, it can be inferred that Chain_1 is classified as Contagion_Chain Unisex, as illustrated in Figure 6c, thereby responding to CQ 4 .

Since it was defined that Patient_3 belongs to the class Patient_Deceased, as shown in Figure 5b, the reasoner classifies Chain_1 as Contagion_Chain_Lethal, thereby responding to CQ 4. It was also defined that patients 2 and 3 live in the district Habana_Vieja and Centro_Habana, respectively, as shown in Figures $5 \mathrm{c}$ and $5 \mathrm{e}$. Therefore, as shown in Figure $6 \mathrm{~b}$, Chain_1 is also classified as Contagion_Chain_MultiCity, thereby responding to CQ 5.

The chains that include members that belong to the class Child are automatically classified as Contagion_Chain_Pediatric. For example, it was defined that Patient_7 is a child, hence, as shows Figure 6d, Chain_2 is classified as Contagion_Chain_Pediatric because it includes Patient_7, thereby responding to CQ 6. As explained above, an extensive transmission chain has at least 5 patients. Therefore, since Chain_2 has 5 members (Figure 4), it is also classified as Contagion_Chain_Large, which answers CQ 7.

If all members of a chain are asymptomatic, it is classified as Contagion_Chain_Asymptomatic. In this case, as shown in Figure $5 \mathrm{~d}$, it is defined that patients 1, 9, 10 and 11 belong to the class Patient_Asymptomatic. In addition, since they are all Patient_Recovered,Chain_3 is classified as Contagion_Chain_Healthy and Contagion_Chain_Asymptomatic, which responds to CQ 8 and CQ 9. Figure 6e shows these inferences.

During the investigation, a group of chains with interesting behavior was identified. The detection of these chains of contagion would allow a more in-depth analysis to be carried out and for possible causes of that behavior to be was created. Additionally, the chains in which a group of patterns are combined could be the ones that should receive the most attention. For example, a chain that consists of patients of only one sex and where all of them are asymptomatic would require an in-depth study.

In this study, a group of chains that could be interesting has been identified. However, the contribution is not in these chains, but in the exploitation of the benefits of OWL to establish the mechanisms to characterize the chains and verify their capacity for analysis. Therefore, this ontology could be enriched with the definition of other types of chains. For example, it would be of interest to verify the behavior of pregnant women who contract the disease or those who have recently had a child. 
The results of the automated analysis of the contagion chains could contribute to epidemiological work or to drawing up new strategies by governments considering social behavior. For example, it is possible to analyze the source of contagion of children under one year. Then, according to the results, new communication campaigns could be carried out to reinforce awareness about the care of children or to provide good practices to mothers to keep their children out of danger.

Similarly, an indicator that considers the members of a chain and the time elapsed between the first and the last case could be stated. Thus, a chain with a high value in this indicator could reveal that the appropriate actions for containment are not being followed or that the virus is spreading rapidly for some reason. Additionally, it is possible to incorporate useful indicators for epidemiological work; for example, the basic reproductive index of each patient and the average cases of a chain. These analyses could provide inputs to epidemiological work and could be used to evaluate the actions that have been applied.

Although this study has focused mainly on illustrating the potential of the ontological model to find interesting information for the analysis of contagion chains, some mechanisms have also been defined to perform analyses on individual patients. For example, the class Disease was defined, meaning for each patient one can verify which previous diseases they had. Likewise, the class Symptom was defined in order to represent the symptoms that a patient may have. In addition, some specifications were established to represent information regarding the vaccines. The correlation of these concepts is planned for our future work.

On the other hand, this ontological model can be queried by several types of software agents that support OWL to find relevant information. Furthermore, some languages to query the ontology can be exploited, for example SPARQL [29]. The ontology can be downloaded at [30].

\section{Conclusions}

In this work, an ontology developed for representing and analyzing information on COVID-19 infection chains has been described. The way that the expressive richness of OWL is exploited to represent different aspects of the chains of contagions has been illustrated. From this ontological model, an automated analysis of the information can be carried out and inferences of interest can be reached for the follow-up of the disease. The ontology was developed following a solid methodology that helped to ensure its quality. The validation of the ontology confirmed that it is correctly designed, it meets the specifications as a formal-logical system and it satisfies the requirements for which it was created. We illustrated by means of a study case the applicability of this ontological model. This approach could be a useful tool to support epidemiological work, especially in countries where rigorous monitoring of contagion chains has proven to be an effective strategy to contain the virus.

It is not the objective of this work to substitute other approaches. The main objective is to provide the community with a new alternative and to contribute to the struggle against COVID-19. We plan to continue this research plans in two directions. First of all, the ontology will be integrated with others that share concepts regarding epidemiology and diseases, for example Infectious Disease Ontology (IDO) [31] and others [12,32-34]. Then, the development of an information system based on the ontological model will be proposed. Although the motivation for this research is the study of COVID-19, the resulting ontology can be used to represent and analyze the behavior of other transmitted diseases between humans, for example HIV.

Author Contributions: Conceptualization, N.S. and A.V.; methodology, N.S., A.V. and E.V.; software, N.S., E.V. and S.A.A.; validation, A.V., V.S.L. and Y.I.R.; formal analysis, N.S., V.S.L. and Y.I.R.; investigation, N.S., A.V. and E.V.; resources, A.V. and Y.I.R.; data curation, A.V.; writing-original draft preparation, N.S., A.V. and E.V.; writing-review and editing, N.S.; visualization, A.V., S.A.A. and E.V.; supervision, A.V. and Y.I.R.; project administration, N.S. and Y.I.R.; funding acquisition, Y.I.R. All authors have read and agreed to the published version of the manuscript. 
Funding: This research was funded by the grants of the Russian Science Foundation, grant number RSF 22-21-00670, https:/ / rscf.ru/project/22-21-00670/, accessed on 12 November 2021.

Institutional Review Board Statement: Not applicable.

Informed Consent Statement: Not applicable.

Data Availability Statement: Not applicable.

Conflicts of Interest: The authors declare no conflict of interest.

\section{References}

1. Xinga, X.; Zhonga, B.; Luoa, H.; Lic, H.; Wua, H. Ontology for safety risk identification in metro construction. Comput. Ind. 2019, 109, 14-30. [CrossRef]

2. Silega, N.; Noguera, M.; Macias, D. Ontology-based Transformation from CIM to PIM. IEEE Lat. Am. Trans. 2016, 14, 4156-4165. [CrossRef]

3. Yanga, L.; Cormicana, K.; Yub, M. Ontology-based systems engineering: A state-of-the-art review. Comput. Ind. 2019, 111, 148-171. [CrossRef]

4. Vences Nava, R.; Menendez Dominguez, V.H.; Gomez Montalvo, J. A document recommendation system using a documentsimilarity ontology. IEEE Lat. Am. Trans. 2016, 14, 3329-3334. [CrossRef]

5. Freitas, S.; Canedo, E.; Jesus, D. Calculating similarity of curriculum lattes. IEEE Lat. Am. Trans. 2018, 16, 1758-1764. [CrossRef]

6. Silega, N.; Noguera, M. Applying an MDA-based approach for enhancing the validation of business process models. Procedia Comput. Sci. 2021, 184, 761-766. [CrossRef]

7. Keet, C.M.; Grütter, R. Toward a systematic conflict resolution framework for ontologies. J. Biomed. Semant. 2021, 12, 15. [CrossRef]

8. Wang, J.; Li, Z.; Cheng, X.; Hu, H.; Liao, C.; Li, P.; Lu, J.; Chen, Z. Epidemiologic characteristics, transmission chain, and risk factors of severe infection of COVID-19 in Tianjin, a Representative Municipality City of China. Front. Public Health 2020, 8, 198. [CrossRef]

9. Schwarz, S.; Steuber, C.; Krafft, H.; Boehm, K.; Martin, D. Pediatric COVID-19 case with regard to the family infection chain and the psychosocial context. Clin. Case Rep. 2020, 8, 3220-3224. [CrossRef]

10. Cheng, X.; Li, Y.; Zhang, Y.; Lu, J. The characteristics of and responses to the two COVID-19 outbreak waves in Hebei Province of China, January 2020 to February 2021. Epidemiol. Infect. 2021, 149, e212. [CrossRef]

11. Hu, K.; Zhao, Y.; Wang, M.; Zeng, Q.; Wang, X.; Wang, M.; Zheng, Z.; Li, X.; Zhang, Y.; Wang, T. Identification of a super-spreading chain of transmission associated with COVID-19 at the Early Stage of the Disease Outbreak in Wuhan. Arch. Clin. Biomed. Res. 2020, 5, 598-612. [CrossRef]

12. He, Y.; Yu, H.; Ong, E.; Wang, Y.; Liu, Y.; Huffman, A.; Huang, H.-h.; Beverley, J.; Hur, J.; Yang, X. CIDO, a community-based ontology for coronavirus disease knowledge and data integration, sharing, and analysis. Sci. Data 2020, 7, 181. [CrossRef]

13. Dutta, B.; DeBellis, M. Codo: An ontology for collection and analysis of COVID-19 data. In IC3K 2020, Proceedings of the 12th International Joint Conference on Knowledge Discovery, Knowledge Engineering and Knowledge Management, Budapest, Hungary, 2-4 November 2020; SciTePress: Setúbal, Portuga, 2020; pp. 76-85.

14. Pesquita, C.; Ferreira, J.D.; Couto, F.M.; Silva, M.J. The epidemiology ontology: An ontology for the semantic annotation of epidemiological resources. J. Biomed. Semant. 2014, 5, 4. [CrossRef] [PubMed]

15. Hulo, C.; Masson, P.; de Castro, E.; Auchincloss, A.H.; Foulger, R.; Poux, S.; Lomax, J.; Bougueleret, L.; Xenarios, I.; Le Mercier, P. The ins and outs of eukaryotic viruses: Knowledge base and ontology of a viral infection. PLoS ONE 2017, 12, e0171746. [CrossRef] [PubMed]

16. Ricaurte, J.A.B.; Sanabria, J.S.G.; Castellanos, G.C. Ontomastitis: Ontología para la Mastitis Bovina. Ing. E Innovación 2016, 4. Available online: https:/ / revistas.unicordoba.edu.co/index.php/rii/article/view/1183 (accessed on 12 November 2021).

17. Noy, N.F.; McGuinness, D.L. Ontology Development 101: A Guide to Creating Your First Ontology; Stanford Knowledge Systems Laboratory Technical Report KSL-01-05 and Stanford Medical Informatics Technical Report SMI-2001-0880; Stanford University: Satanford, CA, USA, 2001.

18. Yang, C.; Ambayo, H.; De Baets, B.; Kolsteren, P.; Thanintorn, N.; Hawwash, D.; Bouwman, J.; Bronselaer, A.; Pattyn, F.; Lachat, C. An Ontology to Standardize Research Output of Nutritional Epidemiology: From Paper-Based Standards to Linked Content. Nutrients 2019, 11, 1300. [CrossRef]

19. Magumba, M.A.; Nabende, P. An ontology for generalized disease incidence detection on twitter. In Proceedings of the International Conference on Hybrid Artificial Intelligence Systems, La Rioja, Spain, 21-23 June 2017; Springer: Cham, Switzerland, 2017; pp. 38-51.

20. Amith, M.; Fujimoto, K.; Mauldin, R.; Tao, C. Friend of a Friend with Benefits ontology (FOAF+): Extending a social network ontology for public health. BMC Med. Inform. Decis. Mak. 2020, 20, 269. [CrossRef] [PubMed]

21. Slimani, T. Ontology development: A comparing study on tools, languages and formalisms. Indian J. Sci. Technol. 2015, 8, 1-12. [CrossRef]

22. Corcho, O.; Fernández-López, M.; Gómez-Pérez, A. Methodologies, tools and languages for building ontologies. Where is their meeting point? Data Knowl. Eng. 2003, 46, 41-64. [CrossRef] 
23. Musen, M.A. The protégé project: A look back and a look forward. AI Matters 2015, 1, 4-12. [CrossRef]

24. Kumar, M.K. Creation of Dynamic Ontologies for Graphical Representation in User Interface using NeOn in Shodhganga. In Proceedings of the ETD2017 Symposium, Washington, DC, USA, 7-9 August 2017.

25. Kotis, K.I.; Vouros, G.A.; Spiliotopoulos, D. Ontology engineering methodologies for the evolution of living and reused ontologies: Status, trends, findings and recommendations. Knowl. Eng. Rev. 2020, 35, e4. [CrossRef]

26. Suárez-Figueroa, M.C.; Gómez-Pérez, A.; Motta, E.; Gangemi, A. Introduction: Ontology Engineering in a Networked World; Springer: New York, NY, USA, 2012; pp. 1-6. [CrossRef]

27. Sattar, A.; Surin, E.S.M.; Ahmad, M.N.; Ahmad, M.; Mahmood, A.K. Comparative analysis of methodologies for domain ontology development: A systematic review. Int. J. Adv. Comput. Sci. Appl. 2020, 11, 99-108. [CrossRef]

28. Poveda-Villalón, M.; Gómez-Pérez, A.; Suárez-Figueroa, M.C. Oops!(ontology pitfall scanner!): An on-line tool for ontology evaluation. Int. J. Semant. Web Inf. Syst. (IJSWIS) 2014, 10, 7-34. [CrossRef]

29. Calvanese, D.; Cogrel, B.; Komla-Ebri, S.; Kontchakov, R.; Lanti, D.; Rezk, M.; Rodriguez-Muro, M.; Xiao, G. Ontop: Answering SPARQL queries over relational databases. Semant. Web 2017, 8, 471-487. [CrossRef]

30. Ontolology to Represent COVID-19 Contagion Chains. Available online: https://drive.google.com/file/d/1PhnpnRseyTczAySlt 0SFDTxzf1yQutF/view?usp=sharing (accessed on 28 December 2021).

31. Cowell, L.G.; Smith, B. Infectious disease ontology. In Infectious Disease Informatics; Sintchenko, V., Ed.; Springer: New York, NY, USA, 2010; pp. 373-395.

32. Alsudias, L.; Rayson, P. Developing an Arabic Infectious Disease Ontology to Include Non-Standard Terminology. In Proceedings of the 12th Language Resources and Evaluation Conference, Marseille, France, 13-15 May 2020; pp. 4842-4850.

33. Natarajan, Y.; Kannan, S.; Mohanty, S.N. Survey of Various Statistical Numerical and Machine Learning Ontological Models on Infectious Disease Ontology. In Data Analytics in Bioinformatics: A Machine Learning Perspective; Satpathy, R., Choudhury, T., Satpathy, S., Mohanty, S.N., Zhang, X., Eds.; Scrivener Publishing LLC: Beverly, MA, USA, 2021; pp. 431-442.

34. Shen, Y.; Yuan, K.; Chen, D.; Colloc, J.; Yang, M.; Li, Y.; Lei, K. An ontology-driven clinical decision support system (IDDAP) for infectious disease diagnosis and antibiotic prescription. Artif. Intell. Med. 2018, 86, 20-32. [CrossRef] [PubMed] 